\title{
Experimental Investigation of Solid Fuel Combustion Process in a Hybrid Porous Reactor
}

\author{
Vojislav Jovicic ${ }^{1,2}$, Nataliia Fedorova ${ }^{1}$, Ana Zbogar-Rasic ${ }^{1}$, Mario Toledo Torres ${ }^{3}$ and Antonio Delgado ${ }^{1,2}$ \\ 1. Institute of Fluid Mechanics (LSTM), Friedrich-Alexander University of Erlangen-Nuremberg, Erlangen D-91058, Germany \\ 2. Erlangen Graduate School in Advanced Optical Technologies (SAOT), Erlangen D-91058, Germany \\ 3. Department of Mechanical Engineering, Technical University Federico Santa María, Valparaíso 05101, Chile
}

Received: June 05, 2017 / Accepted: June 14, 2017 / Published: September 30, 2017

\begin{abstract}
One of the most significant human-made methane emission sources is the MSW (municipal solid waste), deposited on sanitary landfills and open dumps. Within this work, an alternative MSW treatment concept is presented, which could provide a relatively clean waste/biomass-to-energy transformation. The proposed procedure comprises of a combustion and a gasification (or pyrolysis) step, which are consecutively taking place in a two-stage hybrid porous reactor system. The core of the system is two packed bed reactors, in which solid fuel (waste or biomass) is mixed with inert ceramic particles of similar size. This paper overviews the initial experimental investigation of the combustion step of a hybrid mixture, composed of wood pellets (fuel) and alumina balls (inert ceramic particles) in a $250 \mathrm{~mm}$-high batch reactor. The temperature profile along the reactor, the concentration of CO and the flame front propagation velocity were measured as a function of the ceramic particle size (11 and $20 \mathrm{~mm})$, the inert-to-fuel mass ratio (0:1, 2:1, 3:1) and the airflow rate (30, 42, $60 \mathrm{l} / \mathrm{min})$. Experiments indicate that an increase of the mass ratio of inert-to-fuel material and a decrease of the inert ceramic particles size lead to a decrease of the maximum temperature of the packed hybrid bed. Measured CO concentrations showed strong dependence on the inert ceramic particle size, i.e. the particle size reduction from 20 to $11 \mathrm{~mm}$ resulted in a significant reduction of CO-emission peaks. The maximum flame front propagation velocity of $0.2 \mathrm{~mm} / \mathrm{sec}$ was detected for the airflow of $42 \mathrm{l} / \mathrm{min}$, the particle size of $20 \mathrm{~mm}$ and the mass ratio of 3:1.
\end{abstract}

Key words: Combustion, allothermal gasification, MSW, hybrid filtration combustion, packed bed.

\section{Introduction}

A growth of world population leads to an increased amount of produced waste in general, and of MSW (municipal solid waste) per day in particular. According to the World Bank [1], from the total amount of globally produced MSW, currently estimated to 1.3 billion tons per year, almost $80 \%$ end on the sanitary landfills (47.4\%) and open dumps (32.4\%). This form of MSW is a significant emitter of landfill gases, which is one of the major human-made methane sources [1, 2]. The rest of MSW is recycled (6.8\%), incinerated (4.6\%), openly burned (5.4\%) or

Corresponding author: Vojislav Jovicic, Dipl.-Ing., Head of the Research Division B2, research fields: combustion technology, heat transfer and flows with chemical reactions. disposed in other ways (3.4\%).

Since MSW is a global issue, its treatment and utilization are widely investigated [3, 4]. A conventional approach to waste-to-energy treatment is direct combustion of waste (incineration), which has the goal to recover the energetic value of wastes. Other thermochemical treatment methods, i.e. pyrolysis and gasification, may additionally be used for recovering the chemical value of wastes, i.e. for production of chemicals or secondary fuels [5].

The main advantage of gasification over direct combustion of solid wastes is related to the production of syngas, which is suited for use in different applications [6]. Gasifiers can be classified based on the contact between the fuel and the oxidizing agent to fluidized bed gasifiers [7], packed bed gasifiers [8, 9] 
and plasma gasifiers [10]. Gasification processes can be autothermal, where the heat for the endothermic gasification reaction is produced by combustion of part of the fuel inside the reactor, and allothermal (indirect) gasification, where the heat is being supplied from the external source [11]. The gasification of biomass is still a challenging issue due to the production of $\operatorname{tar}$ [12].

While incineration is mainly performed on a moving grate, gasification and pyrolysis can be conducted in a classical packed bed reactor [5, 6]. The advantages of the packed bed treatment are low investment costs (limited to low capacities), high carbon conversion and high flexibility in terms of fuels $[6,13,14]$. On the other hand, poor mixing and inhomogeneous combustion conditions within the bed [14] limit the application of this reactor type.

If packed bed is made of inert material through which gaseous fuel flows, a specific form of combustion occurs, i.e. porous media or filtration combustion [15, 16]. The presence of solid matrix in the combustion zone provides efficient heat transfer between gas and solid, while the dispersion of the gas flowing through a porous media increases effective diffusion and heat transfer in the gas phase [16]. Heat released in the reaction zone is being transferred to the solid matrix immediately above and below the combustion zone, which provides stability of the combustion process in a wide range of gas velocities, equivalence ratios, and power loads [16, 17]. If part of the inert solid particles are exchanged with uniformly distributed solid fuel particles [17], a hybrid filtration combustion reactor is formed, where solid and gaseous fuels can be treated simultaneously $[17,18]$.

\subsection{Motivation and Goal}

The motivation of the presented work was to investigate an alternative two-stage waste/biomass-to-energy treatment concept, which would consist of a combustion step and a consecutive gasification (or pyrolysis) step in two hybrid packed bed reactors. The scheme of the proposed process is shown in Fig. 1.

The main parts of the proposed concept are two hybrid packed bed reactors, where solid fuel (waste or biomass) is mixed with inert ceramic particles of similar size. The inert particles are heated up during the combustion phase within the first reactor, and are pushed to the second reactor, where e.g. allothermal gasification in the presence of steam can take place. The role of the inert particles is to provide the spatially uniform temperature profile of the desired level within the second reactor. Due to the combustion process, the temperature of the inert particles is expected to reach temperatures as high as $900-1,000^{\circ} \mathrm{C}$, which is enough to disintegrate the tar product of gasification, thus increasing the heating value of the obtained fuel. The

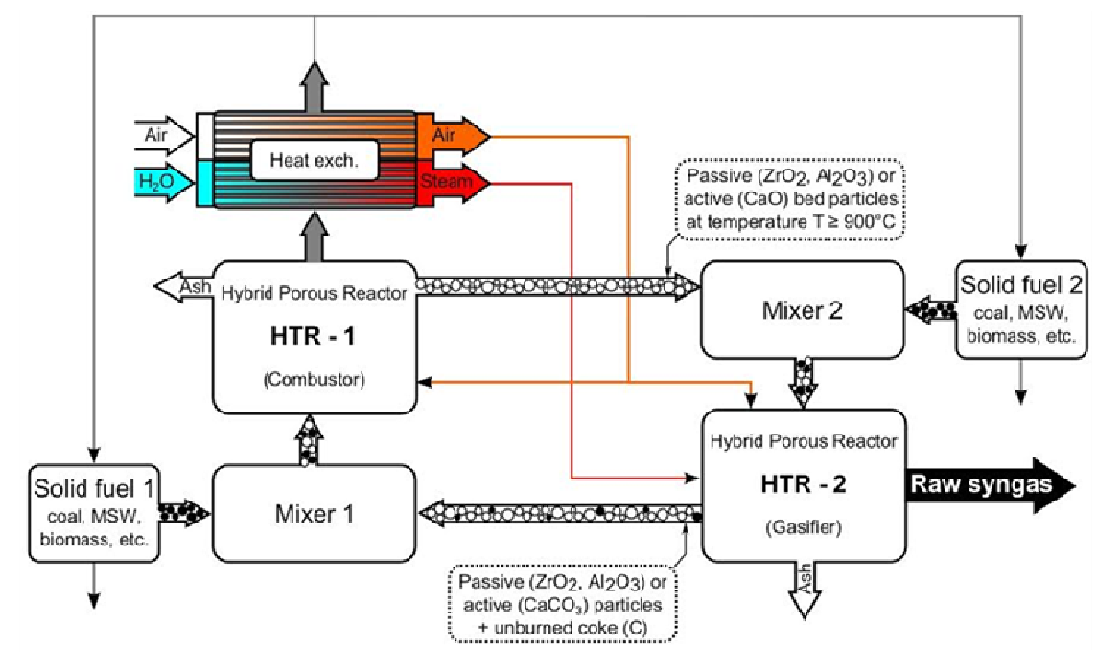

Fig. 1 Scheme of the two-stage hybrid packed bed reactor concept for waste/biomass treatment. 
proposed process would ensure high temperature conditions at the atmospheric pressure, necessary for the production of a high value syngas (composing of $\mathrm{H}_{2}$, $\mathrm{CO}, \mathrm{CH}_{4}$, etc.).

For the successfulness of the gasification (or pyrolysis) step in the second reactor, it is necessary to characterize the operational conditions within the first reactor, especially to determine under which conditions the highest temperature level of inert particles can be reached. According to literature [6, 9], the temperature level required for gasification is between $500{ }^{\circ} \mathrm{C}$ and $1,600{ }^{\circ} \mathrm{C}$, and for pyrolysis between $500{ }^{\circ} \mathrm{C}$ and $800{ }^{\circ} \mathrm{C}$.

The presented study was focused on the combustion process in the first hybrid reactor. The goal was to investigate the influence of the inert particles size, the inert-to-fuel mass ratio and the airflow rate on output parameters, i.e. the temperature distribution along the reactor, the temperature of exhaust gases and the cO-concentration.

\section{Experimental Apparatus and Procedure}

The experimental investigation was focused on the combustion process step, taking place inside a hybrid packed bed reactor. The experimental apparatus, used in this study, is presented in Fig. 2a. The cylindrical batch reactor (Fig. 2b) was filled with a mixture of wood pellets (solid fuel) and alumina balls (inert ceramic particles) (Fig. 2c). The reactor consisted of three parts: (1) air supply, placed at the bottom of the reactor, (2) combustion zone, in the middle of the reactor, and (3) outlet for exhaust gases, at the top of the reactor. The combustion zone, i.e. the packed bed zone, was $250 \mathrm{~mm}$ high, with the inner diameter of 68 $\mathrm{mm}$. An $11 \mathrm{~mm}$ insulation layer was provided for the minimization of heat losses. The packed bed consisted of a randomly premixed wood pellets, with a particle size of $10 \pm 5 \mathrm{~mm}$ (length) $\times 5 \mathrm{~mm}$ (diameter), and inert alumina spheres. The LHV (lower heating value) of the used fuel was $17.64 \mathrm{MJ} / \mathrm{kg}$ (as claimed by the producer).

The flow rate of air, as the oxidant for combustion, was controlled by an MFC (mass flow controller). One set of experiments was conducted with the preheated combustion air, for which purpose an electrical heater was implemented into the air line. The air temperature was measured using a K-type thermocouple (TC5). Four K-type thermocouples (TC1-TC4) were placed at the inner wall (within the insulation), at $60 \mathrm{~mm}$ distance between one another. They measured the temperature distribution along the reactor wall. The CO-concentration in the exhaust gases, which exit the reactor at its top, was quantified by a gas analyzer. An additional K-type thermocouple (TC0) measured the temperature of the exhaust gas. The measured data were collected by a Data Acquisition System, based on the LabVIEW Software.

The ignition took place at the top of the reactor,

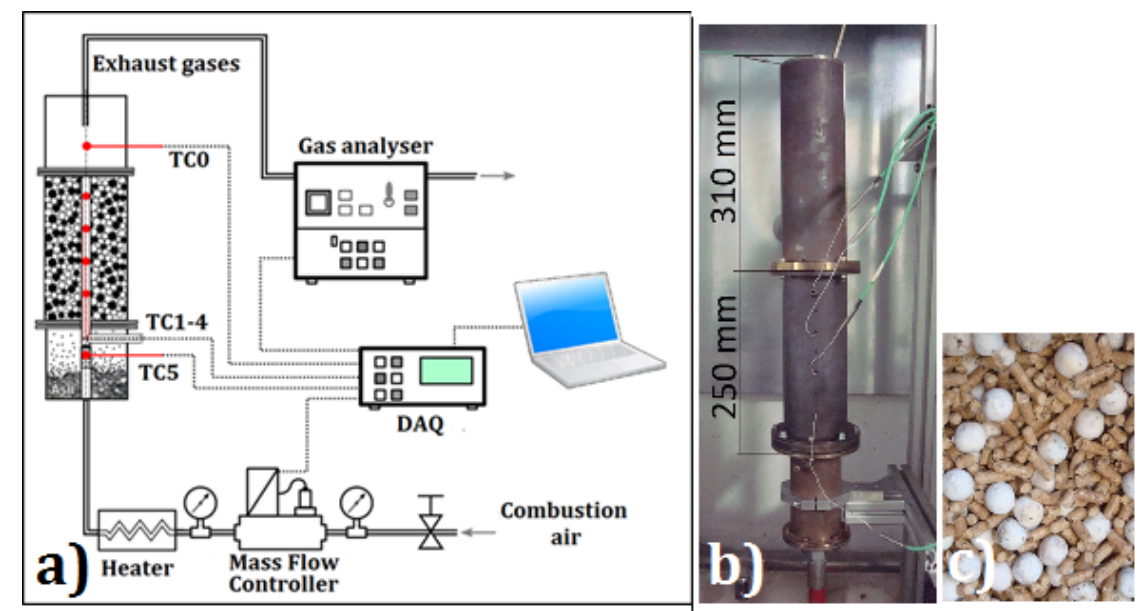

Fig. 2 (a) Scheme of the experimental set-up, (b) hybrid packed bed reactor, and (c) fuel-inert particles mixture. 
using a hand gas burner. Combustion within the reactor was considered to start when TC 0 and TC1 thermocouples experienced a steep temperature increase. As the flame front propagated upstream, the solid fuel was gradually burnt. In this way the transport of alumina particles, left after the solid fuel combustion, took place from top towards the bottom of the reactor.

Depending on experimental conditions, a typical test duration was between 45-75 minutes, which corresponds to the average flame front propagation velocities between 0.092 and $0.055 \mathrm{~mm} / \mathrm{s}$. The end of an experiment was considered when the flame front was at the bottom of the reaction zone, i.e., it was determined based on the temperature measurements.

\section{Results and Discussion}

In the scope of the experimental campaign the following inlet parameters were varied: (1) the inert particles size (diameter $d_{\mathrm{Al}}=11 \mathrm{~mm}$ and $d_{\mathrm{Al}}=20 \mathrm{~mm}$ ), (2) the inert-to-fuel mass ratio $(0: 1,2: 1,3: 1)$, and (3) the air flow rate (30, 42 and $60 \mathrm{~L} / \mathrm{min})$. In order to characterize the combustion in a hybrid reactor, the following output parameters were measured: (1) the temperature distribution along the reactor, (2) the temperature of the exhaust gas, and (3) the
CO-concentration. Experiments showed that the combustion was unstable at the air flow rate of $60 \mathrm{l} / \mathrm{min}$, i.e., the attenuation of the flame and an increased amount of unburned pellets occurred.

Once ignited, the flame front propagated upstream of the reactor, progressively passing each thermocouple from the top (TC1) to the bottom (TC4). Fig. 3 shows an example of the movement of the reaction zone through the reactor, for the inert particles diameter of $d$ $=20 \mathrm{~mm}$, inert-to-fuel mass ratio 3:1, and the volume flow of air $30 \mathrm{~L} / \mathrm{min}$. A steep temperature gradient and temperature peaks correspond to the onset of combustion at different parts of the reactor.

The highest local temperature of ca. $900{ }^{\circ} \mathrm{C}$ occurred in the region of the thermocouple TC3 (placed $105 \mathrm{~mm}$ above the packed bed bottom). In fact, the highest temperatures were recorded in the middle region of the reactor for all the experiments with airflows of 30 and $42 \mathrm{l} / \mathrm{min}$, while for $60 \mathrm{l} / \mathrm{min}$ combustion started to be unstable, and the temperature in the middle zone of the reactor was decreased. The position of the maximum temperature zone in the middle of the reactor could be attributed to decreased heat losses in the middle of the reactor, and to the optimal air/fuel ratio, which is reached in this zone. The lowest temperatures were

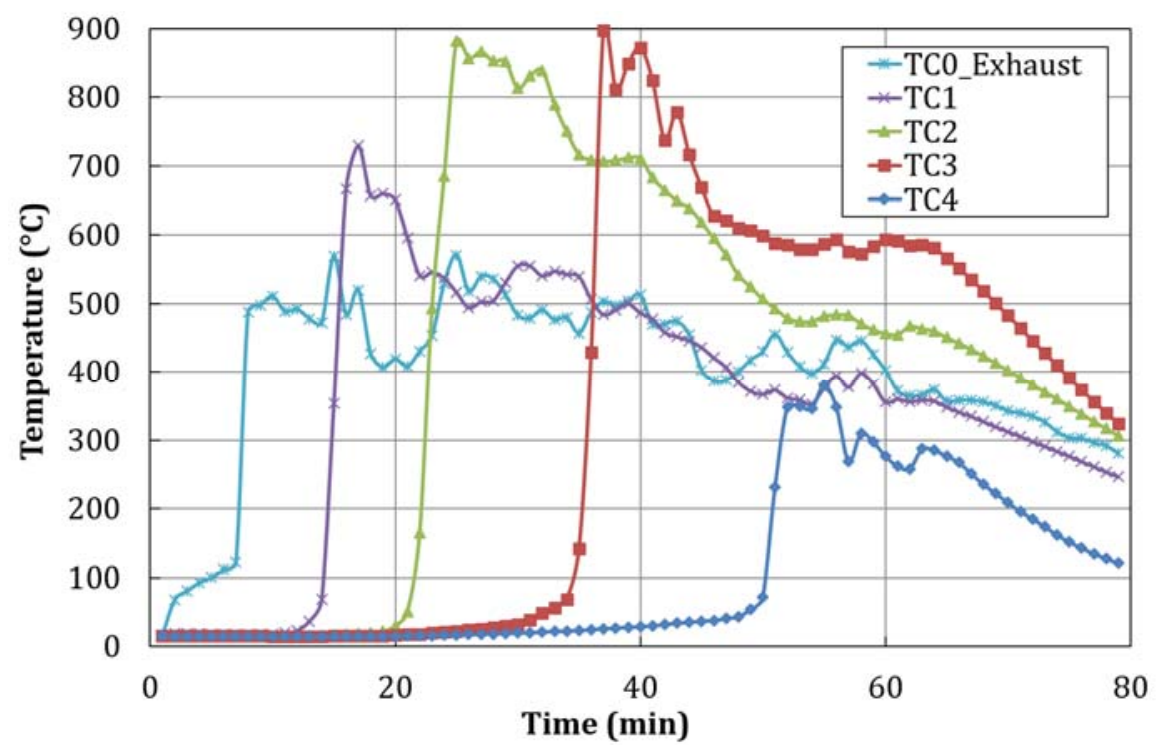

Fig. 3 The time dependence of the temperature profile along the reactor (inert particles diameter $d=20 \mathrm{~mm}$, inert-to-fuel mass ratio 3:1, volume flow of air $30 \mathrm{l} / \mathrm{min}$ ). 
measured at the bottom region of the reactor, because of the cooling effect of the air inflow at the ambient temperature and the consequent high air/fuel ratio.

Temperature profiles showed the similar trend for all conducted experiments. They resembled the temperature profiles of solid material within the filtration combustion reactor, as reported by Toledo [17].

The dependency of the maximum measured temperature on the air flow for all conducted experiments is presented in Fig. 4. At higher inert-to-fuel mass ratios, the peak temperatures were lower, as more inert particles had to be heated. A decrease of the inert particle size led to a decrease of the maximum measured temperature, due to the increased specific surface of the inert particles. The maximum recorded temperature in the reactor at TC3 of $1,064{ }^{\circ} \mathrm{C}$ was reached under the following conditions: the diameter of inert particles $d=20 \mathrm{~mm}$, the inert-to-fuel mass ratio 2:1, and the air flow rate of $42 \mathrm{~L} / \mathrm{min}$. For $60 \mathrm{~L} / \mathrm{min}$ combustion became unstable, as was mentioned before, and the flame front did not reach the thermocouple TC3. Thus, the recorded temperature corresponded to the unburned particles. Based on the results presented in Fig. 4, it can be concluded that the temperature level of inert particles within the combustion reactor is high enough to enable gasification (or pyrolysis) [6] inside the second hybrid reactor.

The flame front propagation velocities were calculated based on the temperature profiles, as is the one shown in Fig. 3. They were determined by detecting the time interval, required for a temperature peak to propagate between two thermocouples (known distance). The dependency of the calculated average flame front propagation velocity on the air flow rate is presented in Fig. 5. Experiments demonstrate that at higher inert-to-fuel mass ratios, the average flame front propagation velocity is lower. A reduction of the particle size at the constant mass ratio results in a decrease of the average flame front propagation velocity.

Obtained results, as shown in Fig. 6, indicated that besides on the air flow rate, the flame front propagation velocity also depends on the position within the reactor. This is influenced by an uneven air distribution within the reactor, i.e., air is consumed in the reactor, and its concentration decreases toward the top of the reactor. The maximum flame front propagation velocity of 0.2 $\mathrm{mm} / \mathrm{s}$ was detected at the top region of the reactor (between thermocouples TC1 and TC2), at the air flow rate of $42 \mathrm{l} / \mathrm{min}$, the inert particle size diameter $d=20$ $\mathrm{mm}$ and the inert-to-fuel mass ratio of 3:1.

During the experiments, high CO emissions above

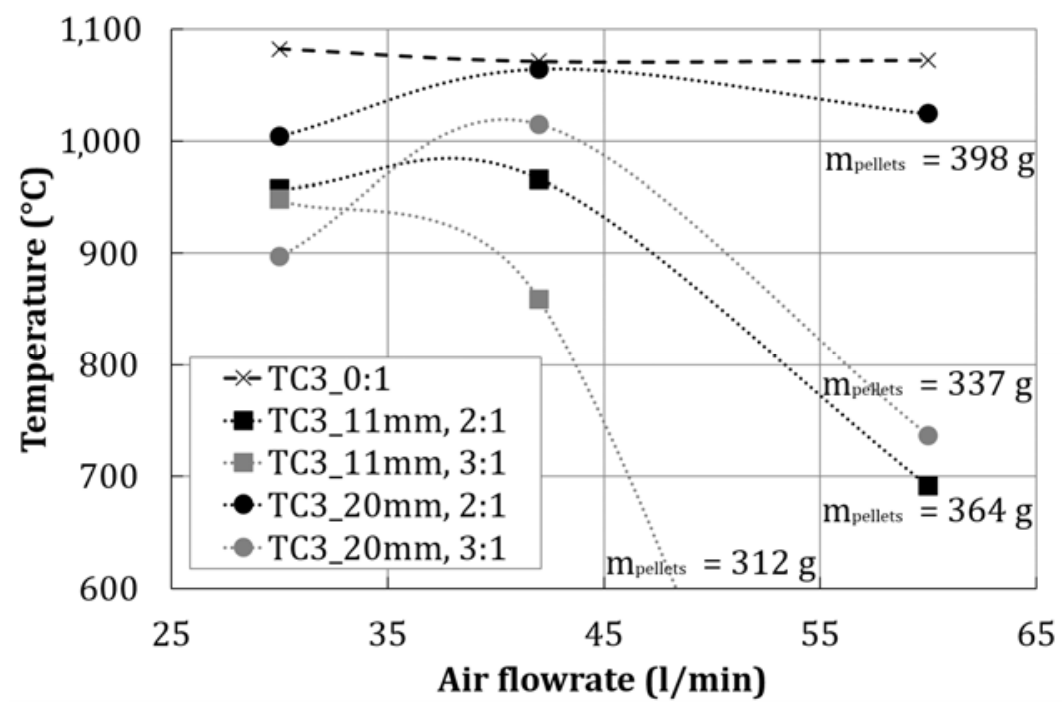

Fig. 4 The dependency of the maximum temperature (position TC3) on the air flow rate for different operating conditions. 


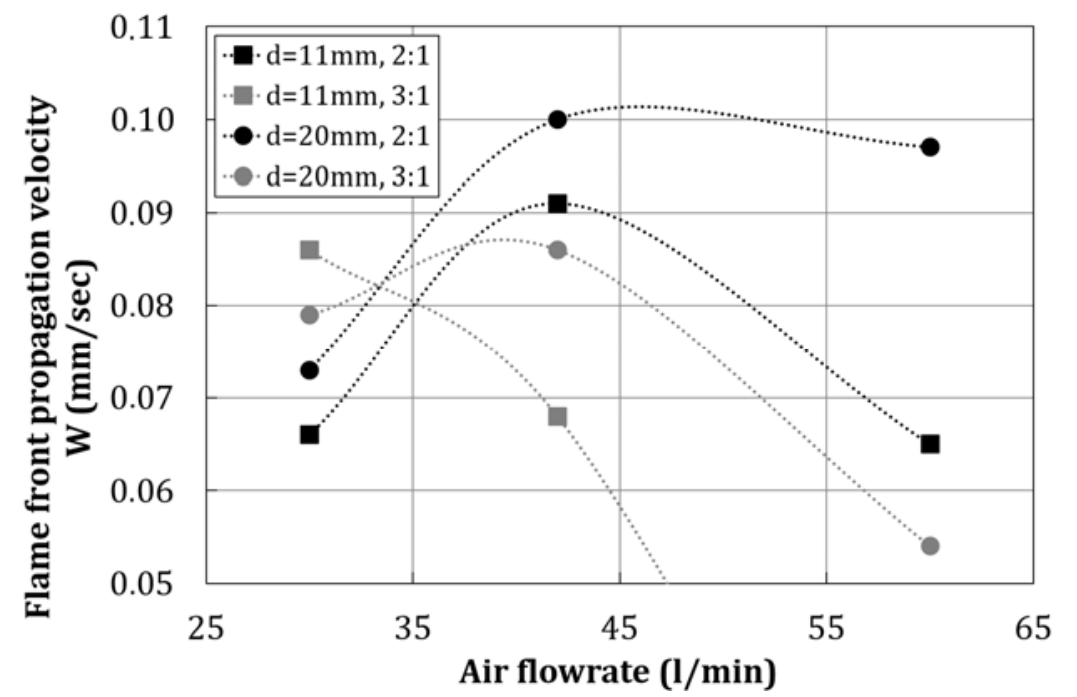

Fig. 5 Dependency of the average flame front propagation velocity on the air flow rate.

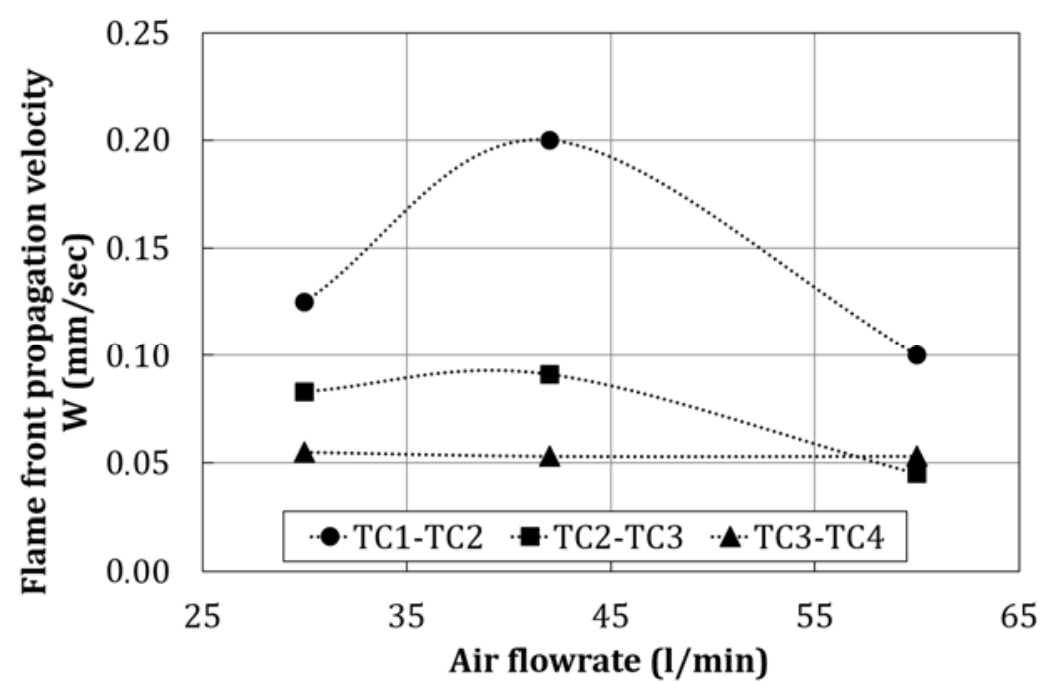

Fig. 6 The flame front propagation velocity at different locations along the reactor at different air flow rates for the particle diameter $d=20 \mathrm{~mm}$, and the mass ratio of $3: 1$.

$1 \%$ were detected, which can be contributed to the poor mixing quality of the oxidant with the fuel particles. As could be expected, lower CO emissions were detected with the increase of air supply and at higher mass ratio (less wood pellets quantity). Measured CO concentrations showed a strong dependence on the inert ceramic particle size. Reduction of the particle size from $20 \mathrm{~mm}$ to $11 \mathrm{~mm}$ resulted in significant reduction of $\mathrm{CO}$ emission peaks, i.e., three to eight times lower.

An additional set of experiments with PCA (preheated combustion air) was performed, for the case when a significant amount of unburned wood pellets was detected, i.e., at the air flow rate of $60 \mathrm{l} / \mathrm{min}$. At the air flow rate of $60 \mathrm{l} / \mathrm{min}$ without the air preheating, the flame could not be stabilized. Thus, the combustion air was preheated from the ambient temperature to $t_{\mathrm{PA}}=$ $150{ }^{\circ} \mathrm{C}$ by an electrical heater. The comparison with the temperature profiles without and with the preheated air is presented in Fig. 7.

Without preheating, the flame extinguished before reaching TC3 (red line without dots) and unburned wood pellets were observed. Preheating of the combustion air enabled the elimination of cold spots within the reactor, stabilization of the combustion process and minimization of the unburned pellets. 


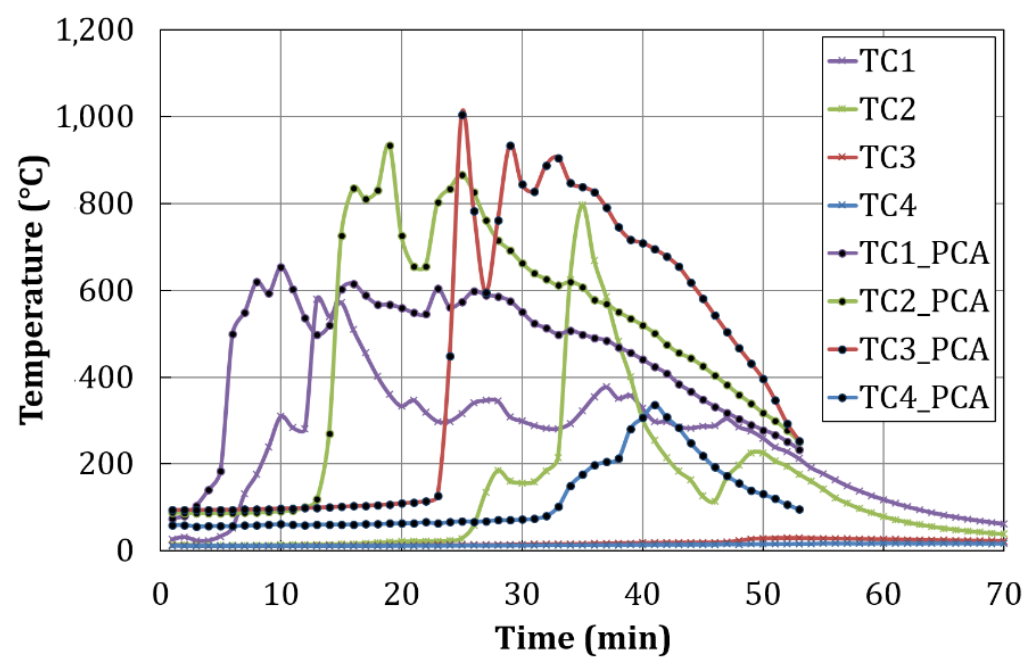

Fig. 7 The time dependency of the temperature distribution along the reactor for the combustion air at the ambient and the elevated temperature.

\section{Conclusions}

Within this study a potential waste/ biomass-to-energy treatment concept was introduced, based on an alternative packed bed treatment, i.e., hybrid filtration combustion. The proposed concept comprises of two steps: combustion step and the gasification (or pyrolysis) step, occurring within two hybrid bed reactors, where fuel particles are randomly mixed with the inert particles of similar size. In order to successfully conduct the gasification (or pyrolysis) step within the second reactor, the combustion process in a hybrid porous bed needs to be characterized.

An experimental characterization of the combustion process in a batch hybrid reactor, filled with a mixture of alumina balls and the wood pellets, was presented. The study included investigation of the impact of the main operating parameters, i.e., the inert particle size, the inert-to-fuel mass ratio and the air flow rate, on the temperature distribution along the reactor, the flame propagating velocity and the concentration of $\mathrm{CO}$.

Experimental results revealed that the highest temperature zone was positioned in the middle region of the reactor. The maximum recorded temperatures, in the range of $950-1,050^{\circ} \mathrm{C}$, were obtained for air flow rates of $30 \mathrm{l} / \mathrm{min}$ and $42 \mathrm{l} / \mathrm{min}$, while at the air flow rate of $60 \mathrm{l} / \mathrm{min}$ the combustion could not be stabilized without air preheating. An increase of the inert-to-fuel mass ratio and a decrease of the inert particles size led to a decrease of the maximum measured temperatures within the reactor. The average calculated flame front propagation velocity along the reactor showed to be lower at higher inert-to-fuel mass ratios and at smaller inert particle size. The maximum determined flame front velocity of $0.2 \mathrm{~mm} / \mathrm{s}$ was reached at the top region of the reactor, at the air flow rate of $42 \mathrm{l} / \mathrm{min}$, with the inert particle diameter $d=20 \mathrm{~mm}$ and at the inert-to-fuel mass ratio of 3:1. Conducted experiments confirmed that inert particles reach the temperature level high enough to provide gasification (or pyrolysis) in the second stage of the hybrid porous reactor.

In order to be able to develop the presented concept, the influence of the particle diameter and the inert-to-fuel mass ratio on the $\mathrm{CO}$-concentration should be further investigated. Numerical analysis of e.g., the influence of the particle size on the air flow pattern through the packed bed, could help in better understanding of the proposed reactor and the concept optimization. Finally, investigation of the gasification (or pyrolysis) step within the hybrid porous reactor needs to be conducted.

\section{Acknowledgements}

The authors gratefully acknowledge Professor Klaus 
Riedle-Stiftung for the financial support and the funding of the Erlangen Graduate School in Advanced Optical Technologies (SAOT) by the German Research Foundation (DFG), in the framework of the German excellence initiative.

\section{References}

[1] Hoornweg, D., and Bhada-Tata, P. 2012. "What a Waste: A Global Review of Solid Waste Management.” Urban development series, knowledge papers 2012; 15, Washington: World Bank. https://openknowledge.worldbank.org/handle/10986/1738 8.

[2] Matthews, E., and Themelis, N. J. 2007. "Potential for Reducing Global Methane Emissions from Landfills.” Presented at the 11th Int. Waste Management and Landfill Symposium, Sardinia.

[3] Gug, J., Cacciola, D., and Sobkowicz, M. J. 2015. "Processing and Properties of a Solid Energy Fuel from Municipal Solid Waste (MSW) and Recycled Plastics.” Waste Management 35 (January): 283-92.

[4] Shareefdeen, Z., Elkamel, A., and Tse, S. 2015. "Review of Current Technologies Used in Municipal Solid Waste-to-Energy Facilities in Canada.” Clean Technologies and Environmental Policy 17 (7): 1837-46.

[5] Helsen, L., and Bosmans, A. 2010. "Waste-to-Energy through Thermochemical Processes: Matching Waste with Process.” Presented at the 1st Int. Symposium on Enhanced Landfill Mining, Houthalen-Helchteren.

[6] Arena, U. 2012. "Process and Technological Aspects of Municipal Solid Waste Gasification: A Review.” Waste Management 32 (4): 625-39.

[7] Arena, U., and Di Gregorio, F. 2014. "Gasification of a Solid Recovered Fuel in a Pilot Scale Fluidized Bed Reactor.” Fuel 17 (1): 528-36.

[8] Khodaei, H., Al-Abdeli, Y., Guzzomi, F., and Yeoh, G. H. 2015. "An Overview of Processes and Considerations in the Modelling of Fixed-Bed Biomass Combustion.” Energy 88 (August): 946-72.

[9] Beenackers, A. A. C. M. 1999. "Biomass Gasification in Moving Beds: A Review of European Technologies." Renewable Energy 16 (1-4): 1180-6.

[10] Zhang, Q., Dor, L., Fenigshtein, D., Yang, W., and Blasiak, W. 2012. "Gasification of Municipal Solid Waste in the Plasma Gasification Melting Process.” Applied Energy 90 (1): 106-12.

[11] Karl, J. 2006. Dezentrale Energiesysteme: Neue Technologien im Liberalisierten Energiemarkt. Oldenbourg Wissenschaftsverlag.

[12] Pereira, E. G., da Silva, J. D., de Oliveira, J. R., and Machado, C. S. 2012. "Sustainable Energy: A Review of Gasification Technologies.” Renewable and Sustainable Energy Reviews 16 (7): 4753-62.

[13] Lackner, M., Palotás, Á., and Winter, F. 2013. Combustion: From Basics to Application. Hoboken: John Wiley \& Sons.

[14] Hester, R. E., and Harrison, R. M. 2013. Waste as a Resource. Royal Society of Chemistry.

[15] Torres M. T., and Huerta, C. R. 2012. "Hybrid Filtration Combustion.” In Hydrogen Energy-Challenges and Perspectives. Croatia: In-Tech.

[16] Mößbauer, S., Pickenäcker, O., Pickenäcker, K., and Trimis, D. 1999. "Application of the Porous Burner Technology in Energy- and Heat-Engineering.” Presented at the Fifth International Conference on Technologies and Combustion for a Clean Environment, Lisbon (Portugal).

[17] Toledo, M., Araus, K., and Vasconcelo, D. 2015. "Syngas Production from Coal in Presence of Steam Using Filtration Combustion." International Journal of Hydrogen Energy 40 (19): 6340-5.

[18] Caro, S., Torres, D., and Toledo, M. 2015. "Syngas Production from Residual Biomass of Forestry and Cereal Plantations Using Hybrid Filtration Combustion.” International Journal of Hydrogen Energy 40 (6): 2568-77. 